\title{
Mineração
}

\section{Cokrigagem colocada aplicada ao mapeamento do nível de água subterrânea}

\author{
Maria Noel Morales Boezio \\ Doutoranda LPM/DEMIN/UFRGS.E-mail:maria.morales@ufrgs.br \\ João Felipe C. L. Costa \\ Professor, Dr.LPM/DEMIN/UFRGS.E-mail: jfelipe@ufrgs.br \\ Jair C. Koppe \\ Professor, Dr.LPM/DEMIN/UFRGS.E-mail: jkoppe@ufrgs.br
}

\section{Resumo}

A mineração subterrânea pode afetar o ciclo hidrológico, especialmente a circulação da água de infiltração de uma dada região. Em particular, o nível de água pode ser modificado, refletindo-se no meio ambiente circundante. Em programas de monitoramento de água subterrânea, o mapeamento do nível de água é parte fundamental. O mesmo ajuda na determinação da direção de fluxo da água subterrânea, identificação de zonas de recarga e avaliação da dispersão de contaminantes. A posição do nível de água subterrânea apresenta correlação, entre outros fatores, com a topografia da superfície, informação de fácil aquisição e baixo custo. Nesse trabalho, apresenta-se a cokrigagem colocada como uma técnica vantajosa para o mapeamento do nível de água, combinando leituras em piezômetros com a informação densamente conhecida da topografia. A metodologia é ilustrada por estudo de caso em uma mina subterrânea de carvão.

Palavras-chave: geoestatística, cokrigagem colocada, modelo linear de corregionalização.

\begin{abstract}
Underground mining can affect the hydrological cycle, mainly groundwater flow. Particularly, water table can be modified, affecting the surrounding environment. In groundwater monitoring programs, water table mapping is one of most important issues. It helps in determining groundwater flow direction, identifying recharge and discharge zones and in evaluating contaminants dispersion. Water table position is related, among other factors, with the surface topography, easily obtainable data at low cost. In this study collocated cokriging is presented as an appropriate procedure to combine exhaustively known topographical information with water level readings at piezometers to map the water table. A case study in an underground coal mine illustrates the methodology.
\end{abstract}

Key words: geostatistics, collocated cokriging, linear model of coregionalization. 


\section{Introdução}

Nas investigações geológicas, são amostradas múltiplas variáveis além daquela que quantifica o principal fenômeno geológico estudado. Se essas variáveis apresentarem correlação com a variável principal (de interesse), o atributo de interesse pode ser estimado a partir de medidas dele próprio (informação primária) conjuntamente com medidas desses outros atributos (informação secundária). No caso do nível de água e topografia, fortemente correlacionados, essa metodologia pode ser aplicada (Desbarats, 2002; Hoeksema, 1989; Samper, 1996).

A incorporação da informação secundária pode ser realizada de diversas formas entre as quais estão a krigagem simples com médias locais variáveis, a krigagem com tendência externa e a cokrigagem (Goovaerts, 1997). A cokrigagem fornece um estimador não tendencioso que minimiza a variância (Wackernagel, 1994) estendendo o estimador de krigagem ao caso de múltiplas variáveis. A sua principal vantagem, frente a outros métodos, é a consideração da correlação espacial conjunta entre variáveis. Também reduz o efeito suavizador, por ser a variância de cokrigagem menor que a de krigagem (Isaaks e Srivastava, 1989), gerando modelos mais consistentes com o fenômeno natural estudado. As vantagens da cokrigagem são maiores quando a variável primária está subamostrada em relação à secundária e quando ambas apresentam alta correlação entre si. Mas uma elevada densidade amostral da variável secundária induz a que dados secundários mais próximos ao local onde se estima a variável primária filtrem a influência de dados mais afastados. Surgiu, assim, a cokrigagem colocada (Xu, 1992), na qual é retido só o dado secundário colocado com o local onde se estima a variável primária.

A água subterrânea interfere nas atividades mineiras, podendo gerar dificuldades no seu controle. A racionalização e a regulamentação da utilização da água subterrânea poderão aumentar o problema para as mineradoras, induzin- do-as à adoção de programas de gestão de recursos hídricos e de monitoramento. Nesse contexto, o nível de água subterrânea, medido em piezômetros, constitui-se em uma variável regionalizada (Matheron, 1965) sobre a qual podem ser aplicadas técnicas geoestatísticas. Essas técnicas levam em consideração a continuidade espacial, característica de alguns fenômenos naturais, para estimar a variável de interesse em locais nos quais não foi amostrada, permitindo a geração de modelos e mapas de nível de água subterrânea.

Nesse trabalho, o objetivo é investigar a aplicação da cokrigagem colocada no mapeamento do nível de água, utilizando a topografia como informação secundária. Por meio de um estudo de caso, avalia-se o benefício dessa metodologia comparado com procedimentos convencionais como, por exemplo, krigagem ordinária. Ao mesmo tempo, com a geração de mapas de nível de água, procura-se satisfazer os requerimentos ambientais e auxiliar no entendimento hidrogeológico dentro do contexto de um programa de gestão de recursos hídricos.

\section{Metodologia}

A metodologia empregada é a cokrigagem colocada ordinária (CKCO) dos dados do nível de água subterrânea, utilizando-se a topografia como informação secundária.

A CKCO pressupõe, semelhantemente à cokrigagem ordinária, estacionariedade das variáveis randômicas $\mathrm{Z}_{1}(\mathrm{u})$ e $\mathrm{Z}_{2}(\mathrm{u})$, utilizadas para modelar o fenômeno, numa vizinhança local centrada no local u onde é realizada a estimativa. Assim, a esperança matemática da variável randômica $E\left\{Z_{\mathrm{i}}(\mathrm{u})\right\}$ existe e é considerada constante na vizinhança W(u). Também existe a função de covariância C(h), que é considerada dependente somente do vetor de separação h (Goovaerts, 1997, p. 70).

O estimador de CKCO é apresentado na Equação 1.

$$
\mathrm{Z}_{\mathrm{CKCO} 1}^{*}(\mathrm{u})=\sum_{\alpha 1=1}^{\mathrm{n}_{1}} \lambda_{\alpha 1}^{\mathrm{CKCO}} \cdot \mathrm{Z}_{1}\left(\mathrm{u}_{\alpha 1}\right)+\lambda_{2}^{\mathrm{CKCO}} \cdot\left[\mathrm{Z}_{2}(\mathrm{u})-\mathrm{m}_{2}+\mathrm{m}_{1}\right]
$$

onde:

$\mathrm{Z}_{\mathrm{CKCO1}}^{*}(\mathrm{u})$ é o estimador de CKCO da variável $\mathrm{Z}_{1}(\mathrm{u})$ no local u.

$\sum_{\alpha 1=1}^{n_{1}} \lambda_{\alpha 1}^{C K C O}$ são os pesos de CKCO para os dados $\mathrm{Z}_{1}\left(\mathrm{u}_{\alpha}\right)$.

$\lambda_{2}^{C K C O}$ é o peso de CKCO para o dado da variável secundária no local u.

$\mathrm{Z}_{1}\left(\mathrm{u}_{\mathrm{a} 1}\right)$ são os dados da variável primária em $\mathrm{n}_{1}$ locais $\mathrm{u}_{\alpha 1}$.

$\mathrm{Z}_{2}$ (u) é o dado da variável secundária no local u.

$\mathrm{m}_{1}$ é a média da variável primária $\mathrm{Z}_{1}(\mathrm{u})$.

$\mathrm{m}_{2}$ é a média da variável secundária $\mathrm{Z}_{2}(\mathrm{u})$.

Utiliza-se uma única condição de não tendenciocidade (Goovaerts, 1998) expressa na Equação 2.

$$
\sum_{\alpha 1=1}^{\mathrm{n}_{1}} \lambda_{\alpha 1}^{\mathrm{CKCO}}+\lambda_{2}^{\mathrm{CKCO}}=1
$$


Como o sistema que resolve os pesos de cokrigagem introduz a covariância das variáveis primária e secundária, conjuntamente com a covariância cruzada entre elas, é necessário realizar a modelagem conjunta da variabilidade espacial. No presente trabalho, essa modelagem é realizada utilizando-se o modelo linear de corregionalização (MLC) (Wackernagel, 1994; Goovaerts, 1997), mas existem outros modelos aplicáveis (Almeida, 1993; Journel, 1999; Rivoirard, 2001; Boezio, 2004; Boezio et al., 2005). No MLC, todas as covariâncias diretas e cruzadas são expressas como combinações lineares das mesmas estruturas básicas (Wackernagel, 1994). Para duas variáveis, o MLC pode ser escrito como:

$$
\begin{aligned}
& \gamma_{11}(h)=b_{11}^{I} \cdot \gamma^{I}+b_{11}^{I I} \cdot \gamma^{I I}+\ldots+b_{11}^{K} \cdot \gamma^{K} \\
& \gamma_{12}(h)=b_{12}^{I} \cdot \gamma^{I}+b_{12}^{I I} \cdot \gamma^{I I}+\ldots+b_{12}^{K} \cdot \gamma^{K} \\
& \gamma_{22}(h)=b_{22}^{I} \cdot \gamma^{I}+b_{22}^{I I} \cdot \gamma^{I I}+\ldots+b_{22}^{K} \cdot \gamma^{K}
\end{aligned}
$$

onde:

$\gamma_{i j}(h)$ é o variograma direto $(i=j)$ e cruzado $(i \neq j)$.

$\mathrm{b}^{\mathrm{k}}{ }_{\mathrm{ij}}$ é a contribuição de cada uma das k estruturas na variância total.

$\gamma^{k}(h)$ é cada uma das k estruturas imbricadas de variância unitária.

As condições que asseguram a definição positiva devem ser cumpridas (Goovaerts, 1997, p. 113).

No estudo de caso, as estimativas foram realizadas em suporte pontual, numa malha de 50 x $50 \mathrm{~m}$, para posterior construção de curvas de nível.

Os algoritmos utilizados nas estimativas são pertencentes à biblioteca GSLIB (Deutsch e Journel, 1998), em particular o programa NEWCOKB3D (Xianlin e Journel, 1999). Para a modelagem da corregionalização, utiliza-se o programa ISATIS ${ }^{\circledR}$ (Bleines et al., 2002).

\section{Estudo de caso}

O estudo de caso utiliza dados provenientes do programa de monitoramento de água subterrânea na Mina do Trevo (mina subterrânea de carvão) localizada em Siderópolis, Santa Catarina. O banco de dados está composto por leituras piezométricas mensais, ao longo do ano 2002, e pelo relevo topográfico, na mesma área de aproximadamente 2700 x 1600 metros. O relevo topográfico foi regularizado na malha de estimativa, utilizando-se a técnica do vizinho mais próximo (nearest neighbor) (Davis, 1986).

Nesse trabalho, apresentam-se os dados correspondentes a duas datas do ano 2002 (22 de janeiro e 26 de dezembro), que apresentam diferente quantidade de piezômetros monitorados e, assim, poder-se-á avaliar a influência da quantidade de dados primários nas estimativas. Na Figura 1, é apresentado o mapa de localização dos dados de nível de água e dos dados da cota topográfica regularizados na malha de estimativa de 50 x 50 metros.

A topografia e o nível de água apresentam uma correlação elevada, como é observado nos coeficientes de correlação que acompanham os gráficos de dispersão da Figura 2. Observa-se que a correlação é mais forte até aproximadamente 140 m e que, para valores maiores, os dados do nível de água e da cota topográfica apresentam uma correlação menor. Por tratar-se de um aqüífero livre, tem-se uma movimentação natural da água em direção a cotas topograficamente mais baixas e, portanto, nas regiões mais elevadas, observa-se uma maior separação entre o nível de água e a cota topográfica que explica a menor correlação.

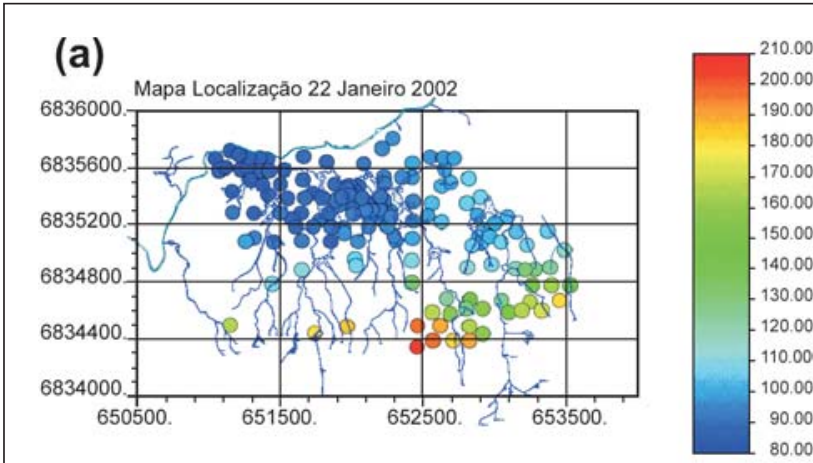

(b)
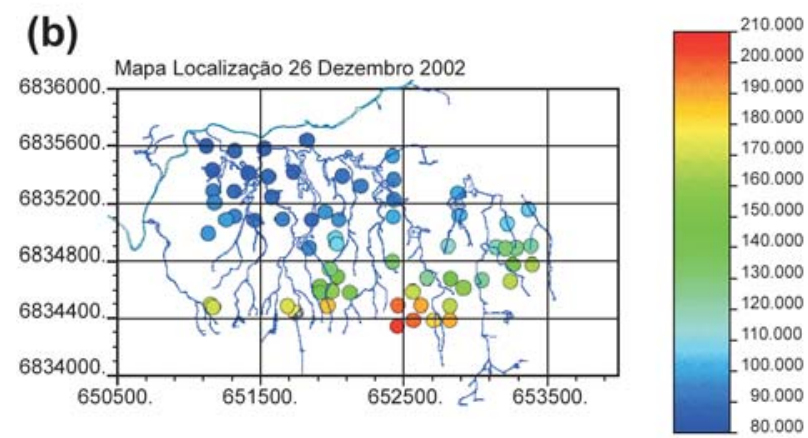

(c)
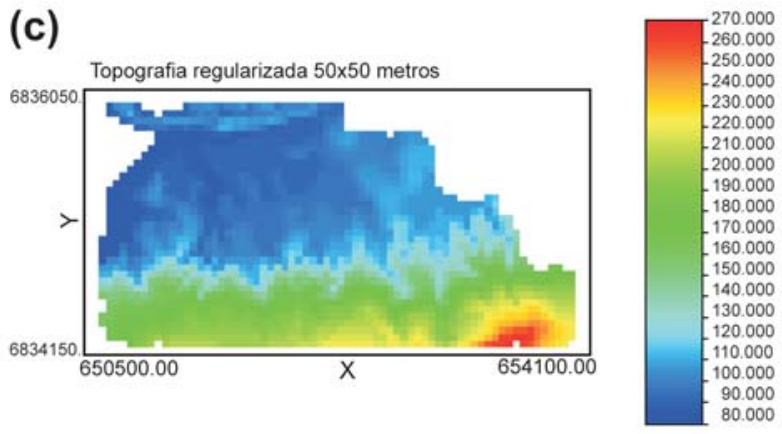

Figura 1 - Mapas de localização dos dados de nível de água (m) obtidos no dia 22 de janeiro (a) e no dia 26 de dezembro de 2002 (b), superpostos aos rios e drenagens da área de interesse; dados da cota topográfica $(\mathrm{m})$ regularizados em malha de $50 \mathrm{x}$ 50 metros(c). 
Os modelos lineares de corregionalização ajustados para os dados da topografia e do nível de água para as duas datas são apresentados na Figura 3.

O conjunto de dados é nitidamente não estacionário, observando-se uma marcada tendência na direção NS. Porém a quantidade de dados é muito abundante e a sua distribuição é densa. Assim, pode ser definida uma vizinhança de busca restrita, na qual pode-se assumir a hipótese de estacionariedade (Journel \& Rossi, 1989). É importante não estender os resultados obtidos no presente estudo para outros casos, onde haja presença de tendência e não se disponha de uma adequada abundância de dados. Nesses casos, seria mais apropriada a utilização de krigagem com tendência externa (Gooaverts, 1997, p.194).
Portanto as estimativas serão realizadas modelando-se os variogramas com anisotropia zonal e utilizando-se uma vizinhança de busca restrita, na qual assume-se estacionariedade. Os mapas das estimativas pontuais do nível de água são apresentados na Figura 4.

\section{Discussão}

Para avaliar a incorporação da topografia como informação secundária analisam-se, comparativamente, as estimativas obtidas por cokrigagem colocada ordinária e por krigagem ordinária realizada, utilizando as mesmas vizinhanças e estratégias de busca. Uma forma de avaliar as estimativas é analisar-se a coerência das mesmas com o fenômeno natural estudado. Para isso, analisa-se a diferença entre a topografia densamente amostrada e o nível de água estimado. Quando essa diferença adquire valores negativos, o nível de água estimado ultrapassaria a cota topográfica, indicando a presença de água. É desejável que a presença de água coincida com locais naturais de ocorrência como drenagens e banhados. Portanto superpõem-se os mapas de rios e drenagens nos mapas das diferenças (Figura 5). A escala na figura indica os valores da diferença.

Nos mapas em que não é utilizada a topografia como informação secundária (KO), é observada uma maior quantidade de locais que indicam a presença de água onde a mesma não é esperada. Isso se observa mais marcadamente no mapa proveniente das estimativas de nível de água por $\mathrm{KO}$ dos dados do dia 26 de

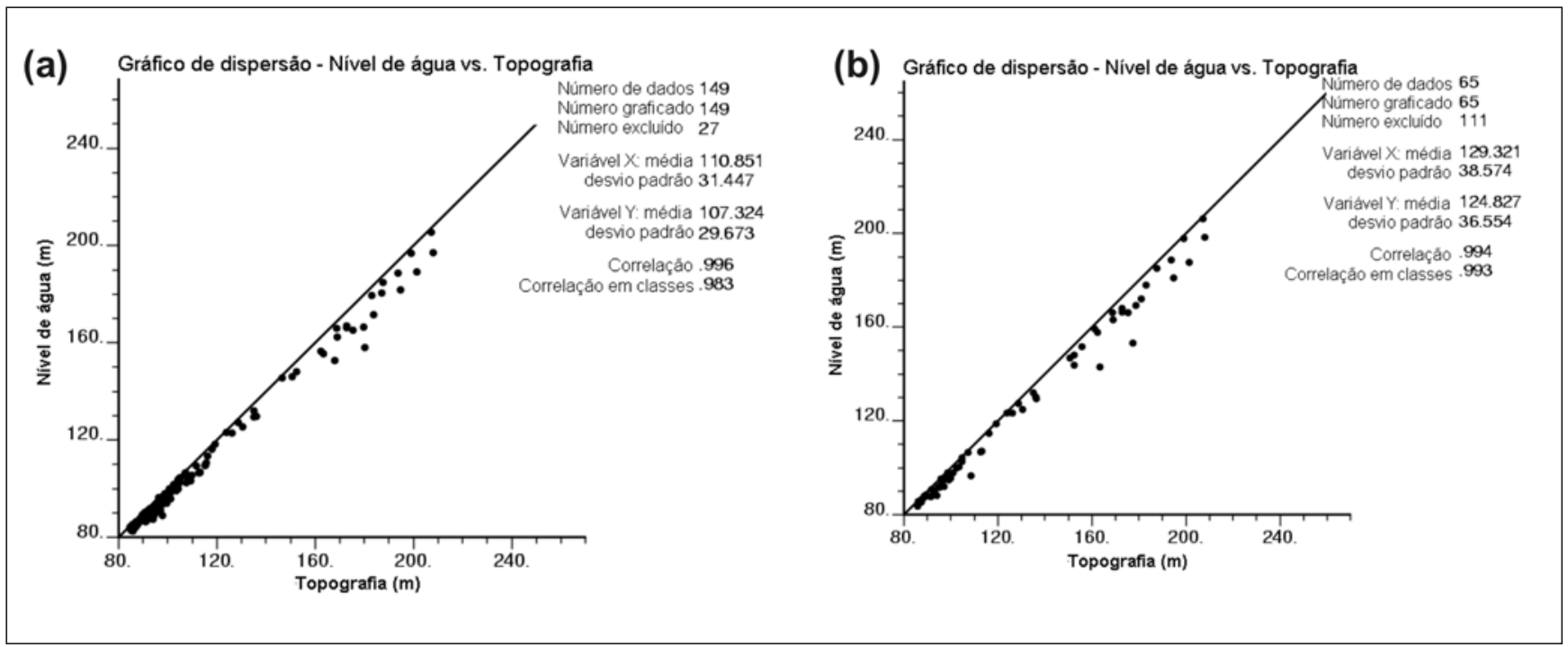

Figura 2 - Gráficos de dispersão dos dados do nível de água (m) vs. cota topográfica $(\mathrm{m})$, mostrando os coeficientes de correlação dos dados do dia 22 de janeiro (a) e do dia 26 de dezembro de 2002 (b).

$$
\begin{aligned}
& \text { (a) } \\
& \gamma_{11}(h)=10+80 \cdot G_{h}\left(\frac{90^{\circ}}{500 m} \frac{0^{\circ}}{470 m}\right)+810 \cdot G_{h}\left(\frac{90^{\circ}}{100000 m} \frac{0^{\circ}}{1080 m}\right) \\
& \gamma_{12}(h)=12+55 \cdot G_{h}\left(\frac{90^{\circ}}{500 m} \frac{0^{\circ}}{470 m}\right)+970 \cdot G_{h}\left(\frac{90^{\circ}}{100000 m} \frac{0^{\circ}}{1080 m}\right) \\
& \gamma_{22}(h)=15+150 \cdot G_{h}\left(\frac{90^{\circ}}{500 m} \frac{0^{\circ}}{470 m}\right)+1857 \cdot G_{h}\left(\frac{90^{\circ}}{100000 m} \frac{0^{\circ}}{1080 m}\right)
\end{aligned}
$$

(b)

$$
\begin{aligned}
& \gamma_{11}(h)=10+190 \cdot G_{h}\left(\frac{90^{\circ}}{400 m} \frac{0^{\circ}}{380 m}\right)+1136 \cdot G_{h}\left(\frac{90^{\circ}}{100000 m} \frac{0^{\circ}}{790 m}\right) \\
& \gamma_{12}(h)=12+135 \cdot G_{h}\left(\frac{90^{\circ}}{400 m} \frac{0^{\circ}}{380 m}\right)+1260 \cdot G_{h}\left(\frac{90^{\circ}}{100000 m} \frac{0^{\circ}}{790 m}\right) \\
& \gamma_{22}(h)=15+150 \cdot G_{h}\left(\frac{90^{\circ}}{400 m} \frac{0^{\circ}}{380 m}\right)+1857 \cdot G_{h}\left(\frac{90^{\circ}}{100000 m} \frac{0^{\circ}}{790 m}\right)
\end{aligned}
$$

Figura 3 - Equações do modelo linear de corregionalização com uma estrutura de efeito pepita e duas estruturas Gaussianas $\left(G_{h}\right)$, ajustado para os dados de cota topográfica e de nível de água correspondentes ao dia 22 de janeiro (a) e ao dia 26 de dezembro (b) de 2002. 
dezembro, onde a abundância de dados é menor. A diferença entre KO e a CKCO é mais evidente nas estimativas provenientes do dia 26 de dezembro. A elevada densidade amostral do nível de água no 22 de janeiro impede que o dado colocado da topografia receba um peso suficiente para diminuir o valor da estimativa do nível de água.

\section{Conclusões}

Depois de aplicar a cokrigagem colocada para o mapeamento do nível de água utilizando a topografia como informação secundária, conclui-se que:

(i) A cokrigagem colocada ordinária mostrou resultados satisfatórios no mapeamento do nível de água sub- terrânea. A utilização da topografia, altamente correlacionada com o nível de água, leva a modelos mais consistentes com o fenômeno natural.

(ii) O melhor resultado obtido no dia 26 de dezembro de 2002, em que a abundância de leituras piezométricas é menor, coincide com as considerações teóricas sobre as vantagens da

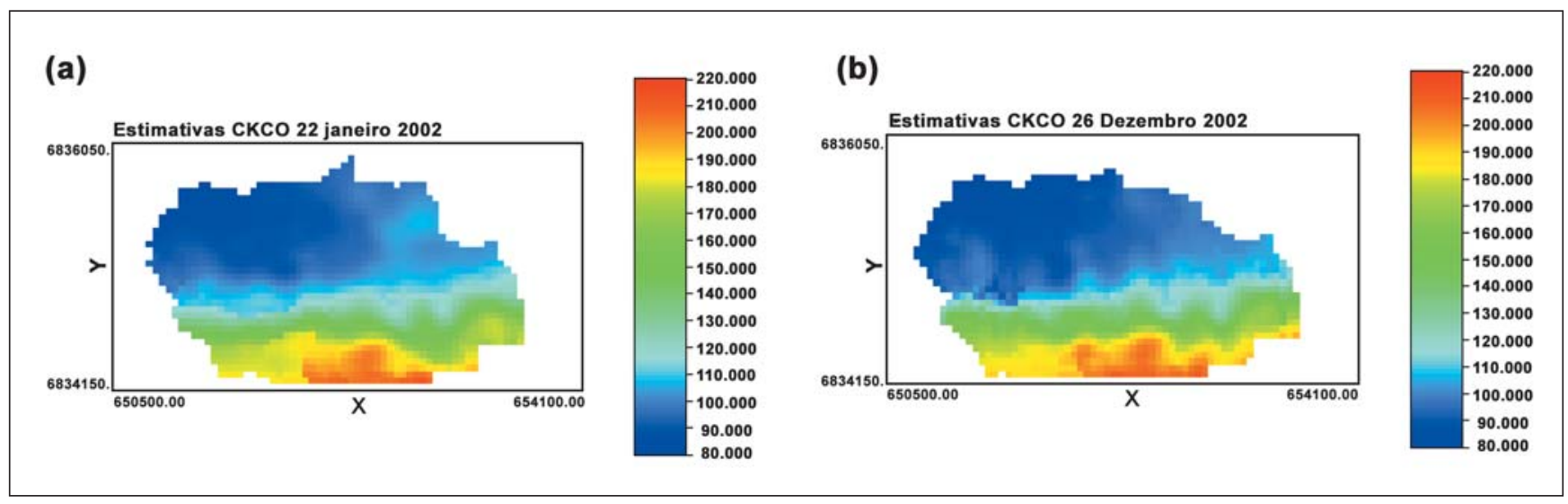

Figura 4 - Estimativas pontuais de nível de água utilizando-se CKCO dos dados de nível de água do dia 22 de janeiro (a) e do dia 26 de dezembro (b) de 2002 e a topografia como informação secundária.

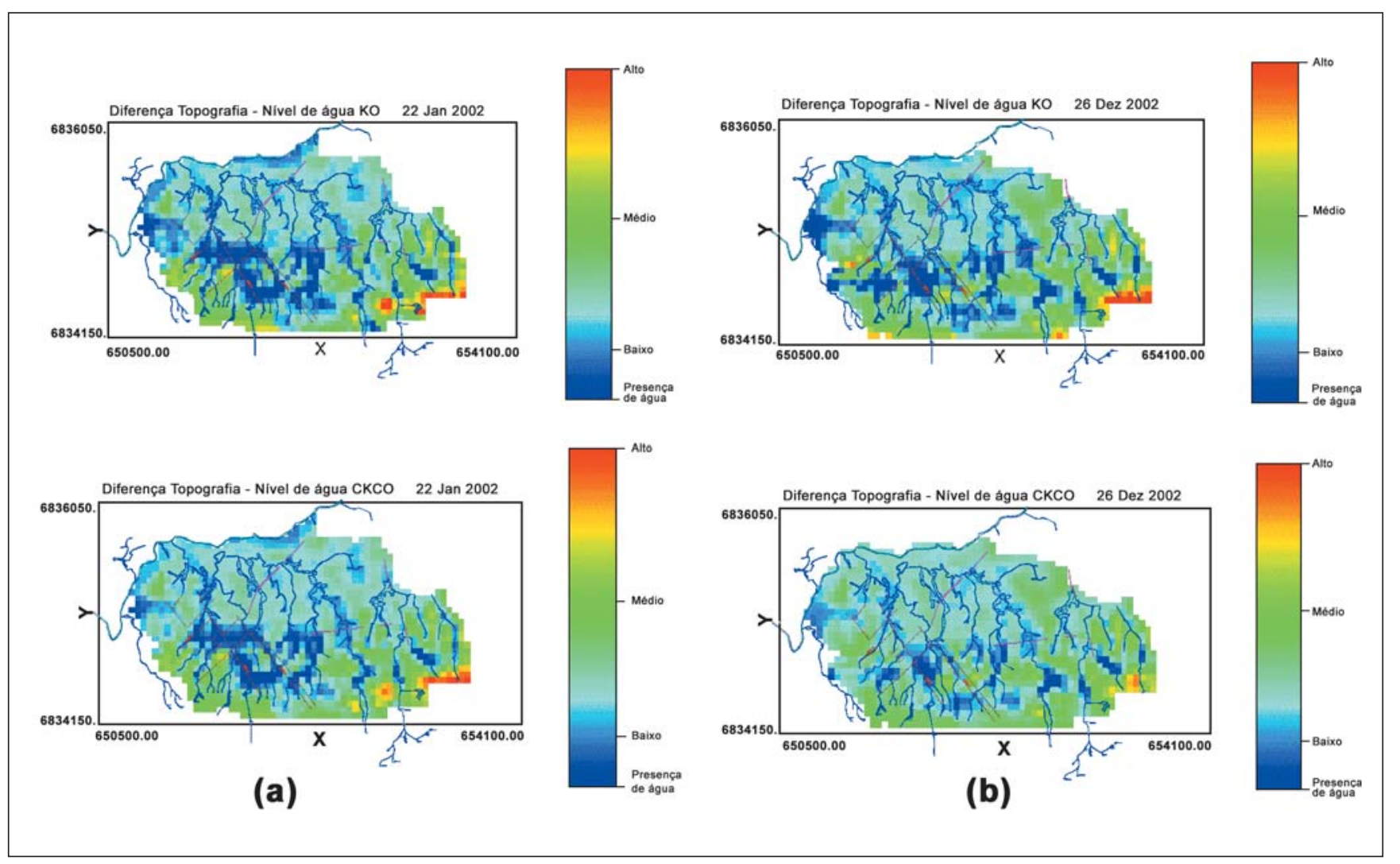

Figura 5 - Mapas da diferença entre a topografia densamente amostrada e o nível de água estimado, utilizando-se krigagem ordinária (OK) e cokrigragem colocada ordinária (CKCO) para o dia 22 de janeiro (a) e para o dia 26 de dezembro (b) de 2002 , superpostos ao mapa de rios e drenagens. 
cokrigagem nos casos em que a variável primária está subamostrada em relação à variável secundária.

(iii) Um número elevado de leituras piezométricas conduz a maiores custos de perfuração e monitoramento. Autilização de uma maior quantidade de piezômetros não fornece mapas de nível de água mais adequados e não permite evidenciar as vantagens da cokrigagem frente a métodos tradicionais devido ao pequeno peso outorgado ao dado secundário colocado. A redução no número de piezômetros junto com a utilização da topografia fornece estimativas mais consistentes.

(iv) Em casos nos quais existe deriva mas não há abundância de dados de forma a utilizar uma vizinhança de busca restrita, é inadequada a utilização da cokrigagem colocada ordinária. Recomenda-se, nessa situação, utilizar a krigagem com tendência externa (KTE). Esse procedimento (KTE), será objeto de publicação futura.

\section{Agradecimentos}

Os autores agradecem à CAPES/ CNPq pela bolsa concedida e às empresas Rio Deserto pela disponibilidade dos dados e demais informações. Aos revisores da REM pelas críticas construtivas que melhoraram a versão desse trabalho.

\section{Referências bibliográficas}

ALMEIDA, A. Joint simulation of multiple variables with a Markovtype coregionalization model.Stanford, California, Estados Unidos: Stanford University, 1993. 199p. (Dissertação de Doutorado).
BOEZIO, M. N. M. Métodos geoestatísticos para a incorporação da topografia como informação secundária no mapeamento do nível de água subterrânea.Porto Alegre: Universidade Federal do Rio Grande do Sul, Brasil, 2004. 292 p. (Dissertação de Mestrado).

BOEZIO, M. N. M., COSTA, J. F. L. C., KOPPE, J. C. Mapping water table Level using piezometers readings and topography as secondary information-Trevo Mine - Brazil. In: INTERNATIONAL SYMPOSIUM ONCOMPUTERAPPLICATIONS IN THE MINERALS INDUSTRY, APCOM 2005-32 ${ }^{\text {nd }}$. Estados Unidos: Universidade de Arizona, 2005. 9p.

BLEINES, C., DERAISME, J., GEFFROY, F., JEANNÉE, N., PERSEVAL, S., RAMBERT, F., RENARD, D., TOUFFAIT, Y. Isatis software manual. (4. ed.). França: Geovariances, Avon, 2002. 645p.

DAVIS, J. C. Statistics and data analysis in geology. New York: John Wiley \& Sons, 1986. 646p.

DESBARATS, A. J., LOGAN, C. E., HINTON, M. J., SHARPE, D. R. On the kriging of water table elevations using collateral information from a digital elevation model. Journal of Hydrology, v. 255, p. 25-38, 2002.

DEUTSCH, C. V., JOURNEL, A. G GSLIB: Geostatistical Software Library and User's Guide. New York: Oxford University Press, 1998. 340p.

GOOVAERTS, P. Geostatistics for natural resources evaluation. New York: Oxford University Press, 1997. 483p.

GOOVAERTS, P. Ordinary Cokriging Revisited. Mathematical Geology, v. 30, n. 1, p. 21-42, 1998.

HOEKSEMA, R. J., CLAPP, R.B. , THOMAS, A. L., HUNLEY, A. E., FARROW, N. D., DEARSTONE, K. C. Cokriging model for estimation of water table elevation. Water Resource Research, v. 25, n. 3, p. 429438, 1989.

ISAAKS, E. H. e SRIVASTAVA, M. R. An introduction to applied geostatistics. New York: Oxford University Press, 1989. 561p.

JOURNEL, A. Markov models for crosscovariances. Mathematical Geology, v.31, n.8, p. 955-964, 1999.

JOURNEL, A., ROSSI, M. E. When do we need a trend model in kriging. Mathematical Geology, v. 21, n. 7, p.715-739, 1989.

MATHERON, G. Les variables regionalisées et leur estimation, Ed. Masson, Paris, 1965, 306p.

RIVOIRARD, J. Which Models for Collocated Cokriging? Mathematical Geology, v. 33, n. 2, p. 117-131, 2001.

SAMPER CALVETE, F. J., CARRERA RAMIREZ, J. Geoestadística. aplicaciones a la hidrogeologia subterranea.(2.ed.) Barcelona: Centro Internacional de Métodos Numéricos en Ingenieria, 1996. 484p.

WACKERNAGEL, H. Multivariate geostatistics. An introduction with applications. Berlim: SpringerVerlag, 1994. 275p.

XIANLIN, M., JOURNEL, A. An expanded GSLIB cokriging program allowing for two Markov models. Computers \& Geosciences, v. 25, p. 627-639, 1999.

XU, W., TRAN, R., SRIVASTAVA, M., JOURNEL, A. Integrating seismic data in reservoir modeling: The collocated cokriging alternative. In: ANNUAL TECHNICAL CONFERENCEOFTHESOCIETY OF PETROLEUM ENGINEERS, 67. Anais... SPE \# 24742, Washington, 1992. p. 833-842.

Artigo recebido em 27/01/2005 e aprovado em 10/10/2005.

\section{REM - Revista Escola de Minas www.rem.com.br}

